\title{
AUTHOR INDEX VOLUME 11 (2001)
}

Abeyratne, U.R., EEG source localization: A compartive study of classical and neural network methods

11 (2001) 349-359

Aler, R., see Galván, I.M.

Almeida, M.B., Training SVMs with EDR algorithm

Andersen, T.L., DMP3: A dynamic multilayer perceptron construction algorithm

Araújo, A.F.R., see Barreto, G. De A.

Avolio, E., see Bordon, M.E.

Barreto, G. De A., Unsupervised learning and temporal context to recall complex robot trajectories

Beigy, H., Backpropagation algorithm adaptation parameters using learning automata Bia, A., Alopex-B: A new, simpler, but yet faster version of the Alopex training algorithm

Bofill, P., Neural cost functions and search strategies for the generation of block designs: An experimental evaluation

Bologna, G., A study on rule extraction from several combined neural networks

Bordon, M.E., see da Silva, I.N.

Bordon, M.E., Design of a neurofuzzy controller with simplified architecture

Bosch, H., see Labbi, A.

Braga, A.P., see Almeida, M.B.

Braga, A.P., see de Albuquerque Teixeira, R.

Braga, J.P., see Almeida, M.B.

Breakspear, M., Perception of odors by a nonlinear model of the Olfactory bulb

Brouwer, R.K., A proposed max-product threshold unit for classification of pattern vectors

Bullinaria, J.A., Neural network control systems that learn to perform appropriately

Canuto, A., Improving ARTMAP learning through variable vigilance

Card, H.C., see McNeill, D.K.

Card, H.C., Stochastic radial basis functions

Cardot, H., see Lezoray, O.

Cavill, S.J., see Vaughn, M.L.

Ceccatto, H.A., see Granitto, P.M.

Chan, L.-W., Weight groupings in second order training methods for recurrent networks

Cheema, T. A., Artificial neural networks for blur identification and restoration of nonlinearly degraded images

Chen, A.-P., Establishing an index arbitrage model by applying neural networks method A case study of Nikkei 225 index

Chianglin, C.-Y., see Chen, A.-P.

Cho, S., Modelling and prediction for chaotic FIR laser attractor using rational function neural network

Cho, S., Neural network based automatic diagnosis of children with brain dysfunction

Chung, H.-P., see Chen, A.-P.

Cosme, J.M.Z., see Lanza, P. A. G.

Coward, A., Application of the recommendation architecture to telecommunications network management

da Silva, I.N., see Bordon, M.E.

da Silva, I.N., A novel approach for solving constrained nonlinear optimization problems using neurofuzzy systems

de Albuquerque Teixeira, R., Recent advances in the MOBJ algorithm for training artifical neural networks

de Carvalho, A., see Lacerda, E.

11 (2001) 167-177

11 (2001) 257-263

11 (2001) 145-165

11 (2001) 11-22

11 (2001) 229-235

11 (2001) 11-22

11 (2001) 219-228

11 (2001) 497-507

11 (2001) 187-202

11 (2001) 247-255

11 (2001) 281-286

11 (2001) 229-235

11 (2001) 371-378

11 (2001) 257-263

11 (2001) 265-270

11 (2001) 257-263

11 (2001) 101-124

11 (2001) 271-279

11 (2001) 79-88

11 (2001) 509-522

11 (2001) 389-398

11 (2001) 203-210

11 (2001) 33-42

11 (2001) 335-347

11 (2001) 305-310

11 (2001) 379-387

11 (2001) 455-461

11 (2001) 489-496

11 (2001) 489-496

$11(2001) 89-99$

11 (2001) 361-369

11 (2001) 489-496

11 (2001) 71-77

11 (2001) 323-327

11 (2001) 229-235

11 (2001) 281-286

11 (2001) 265-270

11 (2001) 287-294 
de Castro, L.N., Automatic determination of radial basis functions: An immunity-based approach

De Moor, B., see Duhoux, M.

de Oliveira, P.P.B., see Santos, C.L.R.

de Sousa Jr., C., Adaptive control of mobile robots using a neural network

de Souza, A.N., see da Silva, I.N.

Diederich, J., see Hogan, J.M.

Duhoux, M., Improved long-term temperature prediction by chaining of neural networks

Fairhurst, M., see Canuto, A.

Fiori, S., Probability density function learning by unsupervised neurons

Fogg, A.J.B., see Vaughn, M.L.

Foy, M.A., see Vaughn, M.L.

Galván, I.M., A selective learning method to improve the generalization of multilayer feedforward neural networks

Gedeon, T., see Coward, A.

Geib, J., see Serpen, G.

Goltsev, A., A recurrent neural network for partitioning of hand drawn characters into strokes of different orientations

Gomide, F. see Romero, R.A.F.

Granitto, P.M., A late-stopping method for optimal aggregation of neural networks

Hemerly, E.M., see de Sousa Jr., C.

Herrero, J.C., CDMA \& TDMA based neural nets

Hévízi, G., see Lőrincz, A.

Hogan, J.M., Recruitment learning of Boolean functions in sparse random networks

Howells, G., see Canuto, A.

Husbands, P., see Santos, C.L.R.

Isasi, P., see Galván, I.M.

Jalil, A., see Cheema, T.A.

Kacpryzk, J. see Romero, R.A.F.

Kawano, H., see Yamakawa, T.

Kenworthy, W., see Coward, A.

Labbi, A., High order statistics for image classification

Lacerda, E., Evolutionary optimization of RBF networks

Lanza, P.A.G., A short-term temperature forecaster based on a novel radial basis functions neural network

Lezoray, O., A neural network architecture for data classification

Lima, P.M.V., A goal-driven neural propositional interpreter

Lörincz, A., Ockham's razor modeling of the matrisome channels of the basal ganglia Thalamocortical loops

Ludermir, T., see Lacerda, E.

Martinez, T.R., see Andersen, T.L.

Martins, J., Neural networks and logical reasoning systems: A translation table

Matsuda, Y., Global mapping analysis: Stochastic approximation for multidimensional scaling

McNeill, D. K., An investigation of competitive learning for autonomous cluster identification in embedded systems

Meesad, P., see Yen, G.G.

Mendes, R.V., see Martins, J.

Meybodi, R., see Beigy, H.

Murray, A., Analogue VLSI for probabilistic networks and spike-time computation

Murray, A.F., see McNeill, D.K.

Naveed, A., see Cheema, T.A.

Navone, H.D., see Granitto, P.M.

Patwardhan, A., see Serpen, G.

Pellegrini, C., see Labbi, A.

Qureshi, I.M., see Cheema, T.A.

Rachkovskij, D., see Goltsev, A.

Riddell, P.M., see Bullinaria, J.A.

Romero, R.A.F., A biologically inspired neural network for dynamic programming

Sakurai, A., A fast and convergent stochastic MLP learning algorithm

Saldanha, R.R., see de Albuquerque Teixeira, R.
11 (2001) 523-535
11 (2001) 1-10
11 (2001) 295-304
11 (2001) 211-218
11 (2001) 281-286
11 (2001) 537-559
11 (2001) 1-10
11 (2001) 509-522
11 (2001) 399-417
11 (2001) 335-347
11 (2001) 335-347
11 (2001) 167-177
11 (2001) 323-327
11 (2001) 477-487
11 (2001) 463-475
11 (2001) 561-572
11 (2001) 305-310
11 (2001) 211-218
11 (2001) 237-246
11 (2001) 125-143
11 (2001) 537-559
11 (2001) 509-522
11 (2001) 295-304
11 (2001) 167-177
11 (2001) 455-461
11 (2001) 561-572
11 (2001) 329-334
11 (2001) 323-327
11 (2001) 371-378
11 (2001) 287-294
11 (2001) 71-77
11 (2001) 33-42
11 (2001) 311-322
11 (2001) 125-143
11 (2001) 287-294
11 (2001) 145-165
11 (2001) 179-186
11 (2001) 419-426
11 (2001) 389-398
11 (2001) 427-443
11 (2001) 179-186
11 (2001) 219-228
11 (2001) 23-32
11 (2001) 389-398
11 (2001) 455-461
11 (2001) 305-310
11 (2001) 477-487
11 (2001) 371-378
11 (2001) 455-461
11 (2001) 463-475
11 (2001) 79-88
11 (2001) 561-572
11 (2001) 573-583
11 (2001) 265-270 
Santos, C.L.R., Three case studies of the gasnet model in discrete domains

Saratchandran, P., see Abeyratne, U.R.

Serpen, G., The simultaneous recurrent neural network for addressing the scaling problem in static optimization

Shin, M.S., see Cho, S.

Souza, C.R., see Santos, C.L.R.

Suykens, J., see Duhoux, M.

Szepesvári, C., see Lőrincz, A.

Szeto, C.-C., see Chan, L.-W.

Takahashi, R.H.C., see de Albuquerque Teixeira, R.

Tambouratzis, T., String matching artificial neural networks

Taylor, S.J., see Vaughn, M.L.

Torras, C., see Bofill, P.

Valls, J.M. see Galván, I.M.

Vandewalle, J., see Duhoux, M.

Vaughn, M.L., Direct explanations for the development and use of a multi-layer perceptron network that classifies low-back-pain patients

Verdes, P.F., see Granitto, P.M.

$\mathrm{Xu}, \mathrm{L} .$, Best harmony, unified RPCL and automated model selection for unsupervised and supervised learning on Gaussian mixtures, three-layer nets and ME-RBF-SVM models

Yamaguchi, K., see Matsuda, Y.

Yamakawa, T., Identification of a landmark in a roentgenographic cephalogram by employing the wavelet neurons

Yen, G.G., Constructing a fuzzy rule-based system using the ILFN network and genetic algorithm

Zhang, G., see Abeyratne, U.R.

Zuben, F.J.V., see de Castro, L.N.
11 (2001) 295-304

11 (2001) 349-359

11 (2001) 477-487

11 (2001) 361-369

11 (2001) 295-304

11 (2001) $1-10$

11 (2001) 125-143

11 (2001) 379-387

11 (2001) 265-270

11 (2001) 445-453

11 (2001) 335-347

11 (2001) 187-202

11 (2001) 167-177

11 (2001) 1-10

11 (2001) 335-347

11 (2001) 305-310

11 (2001) 43-69

11 (2001) 419-426

11 (2001) 329-334

11 (2001) 427-443

11 (2001) 349-359

11 (2001) 523-535 